\title{
Over-the-Air Phase Measurement and Calibration Method for 5G mmW Phased Array Radio Transceiver
}

\author{
Markku Jokinen*, Olli Kursu*, Nuutti Tervo*, Jani Saloranta*, Marko E. Leinonen*, Aarno Pärssinen* \\ ${ }^{*}$ Centre for Wireless Communications, University of Oulu, Finland
}

\begin{abstract}
In this paper, over-the-air (OTA) phase measurement and calibration method for a fifth generation (5G) millimeter wave radio frequency unit is presented. The phase error measurements are done for both receiver and transmitter. The measurement results are used to calibrate the individual antenna ports of the antenna array system so that accurate radiation patterns can be formed. In order to confirm the calibration, the radiation patterns are measured with a $100 \mathrm{MHz}$ wide $28 \mathrm{GHz}$ 5G New Radio communication signal. The measurements show that the calibration improves the maximum power level flatness of radiation patterns and reduces side lobe levels.

Index Terms-beamforming, calibration, phased array, reciprocity, wideband
\end{abstract}

\section{INTRODUCTION}

In fifth generation (5G) communication systems higher capacity is achieved mainly by using wider bandwidths offered by higher carrier frequencies. Several millimeter-wave $(\mathrm{mmW})$ bands are already specified in the standard and one of them is located at around $28 \mathrm{GHz}$ [1]. Compared to the long term evolution (LTE) frequencies, the drawback of $\mathrm{mmW}$ is the higher path loss caused by the decreased physical area of an antenna, the absorption loss of atmospheric gases, and shadowing effect of obstacles [2]. To compensate for the high path loss at $\mathrm{mmW}$, directive antennas can be used to concentrate the radiated energy to or from the transmitted or received direction, respectively. In a mobile scenario, a practical way of generating directive steerable beam is to use phased arrays.

In order to create a directional radiation pattern and to steer the direction of the beam maxima and minima, the phase and amplitude levels of an individual antenna element are required to be controlled with sufficient accuracy. The required accuracy depends on e.g. required main beam width, main lobe gain and side lobe levels [3]. An example of a phased array system that is used without calibration is presented in [4]. This highly integrated implementation uses several radio frequency (RF) integrated circuits (ICs) to drive the antennas.

Systems with high integration rate are mainly suitable for low power applications and hence are applicable for the user equipment (UE). On the contrary, base station (BS) side requires more power. High power offered by discrete component implementations or an array of integrated transceivers is needed especially in systems designed for mobile backhaul such as [5], [6]. In such implementations, small variations between individual $\mathrm{mmW}$ components, on printed circuit board

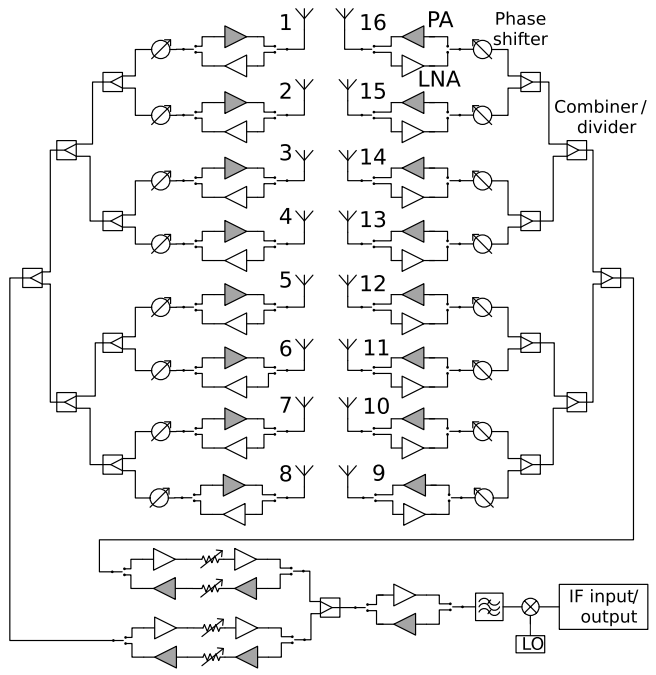

Fig. 1: Simplified block diagram of the RF unit.

(PCB) manufacturing process and component assembly makes the RF paths quite different. Variations in path lengths and in component values make the phase and amplitude response vary over different branches and hence causes errors in beamforming. For these reasons, antenna-specific complex calibration coefficients are required to be measured and applied.

In this work, our focus is on phase measurement and calibration. The phased array unit used in the measurement is presented in Chapter II and the OTA phase measurement setup and calibration procedure is described in Chapter III. The calibrated radiation patterns are validated in Chapter IV by modulated signal measurements.

\section{Calibrated Phased Array Transceiver}

In the previous work [6], a discrete component mmW RF unit that supports eight antenna ports was designed. In this paper, an updated RF unit is used which is capable of driving two times larger antenna array. A simplified block diagram of the $5 \mathrm{G}$ backhaul prototype RF unit is presented in Fig. 1. It is capable of controlling phases of each 16 antenna ports individually by 5-bit phase shifters with $11.25^{\circ}$ steps. Each branch has its own phase shifter shared by the transmitter (TX) and receiver (RX) paths [5]. The TX paths are equipped with Gallium nitride ( $\mathrm{GaN}$ ) power amplifiers (PAs) while the RX paths have Gallium arsenide (GaAs) low noise amplifiers (LNAs). Thus, separate measurements and compensations may be needed for TX and RX paths. 
The phase values for each RF branch are calculated by discrete Fourier transform (DFT) method [7]. It is used to create the beamforming weight-vector to form the radiation pattern. This method creates a progressive phase excitation to the antenna ports [8]. The antenna array has 16 ports that drive a 64-element array [9], i.e. each port is equipped with a $2 \times 2$ antenna sub-array with unitary phase excitation. The sub-arrays are placed in $8 \times 2$ formation.

\section{Wideband OTA Phase Calibration}

\section{A. Conducted vs Radiated Measurements}

The phase errors between the phased array branches may originate from the RF implementation, but also the physical interface used to attach the antenna unit and the antenna implementation itself can be a significant source of phase error. In order to use conducted calibration, the effect of antenna and its interface to the RF unit is required to be measured separately leading to a complex and cumbersome setup. Furthermore, conducted, i.e. cable connected, measurement procedure often requires external calibration of the measurement setup. In addition, it is often challenging to conduct accurate phase measurements over a frequency-converting device.

In order to capture all the inaccuracies of the phased array unit, the measurements are performed over-the-air with a link distance of 4 meters. In such a setup, another array or transceiver can act as a reference unit as long as it is capable of providing the same frequency conversion. In the presented OTA measurement setup, the input and output ports of the measurement device are operating at the same intermediate frequency (IF). Furthermore, OTA is the preferred measurement method for mmW UE and BS units in the 3GPP standard [1] for characterizing the transceiver performance.

\section{B. OTA Phase Measurement Setup}

Measurement setup is presented in Fig. 2. The setup was placed in an anechoic chamber to reduce the external interference. For OTA phase measurement, we used PNA-X network analyzer to measure standard scattering parameters (S-parameters) between the device under test (DUT) and a reference unit. The measurements were conducted over all antenna branches individually by using the transceiver (TRX) switch to enable and disable branches. Calibration of the measurement system itself was not needed due to the fact that the phase measurement is relative. Hence, the effects of cabling, propagation channel and the reference RF unit are cancelled out automatically in the post-processing. In postprocessing, one antenna branch was selected as the reference branch, which was then used to calculate the relative phase of all other branches. Hence, the signal on the reference branch goes through all the same components in the measurement system than the signal on branches we want to analyze, so what remains after comparison to the reference is the phase response of that particular antenna branch. Measurement distance is assumed to be long enough to have on effect on the measured phase. The same setup can be used to measure both RX and TX by changing the operation mode of the DUT

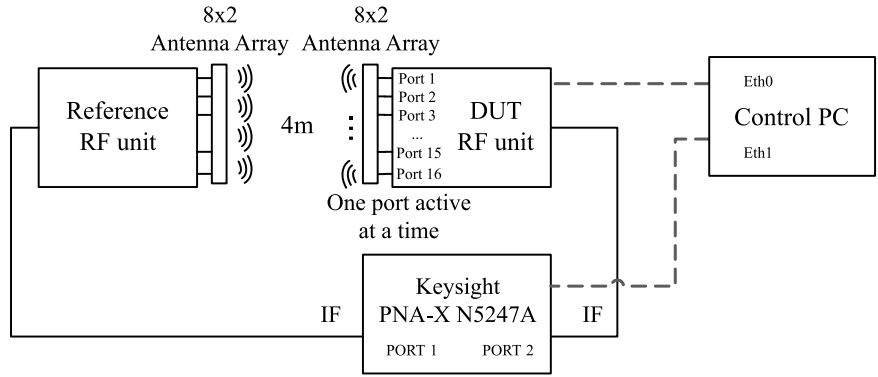

Fig. 2: OTA phase measurement setup.

and reversing the PNA ports. In all the measurements, the equipment and the DUT were synchronized with a $10 \mathrm{MHz}$ reference clock signal.

The measurement follows the so-called virtual or switched array principle [10] used traditionally in channel sounding. The principle measures the individual antenna channels against the same phase and amplitude reference and hence provides the contribution of each antenna element branch to produce overall multiple-input multiple-output (MIMO) channel.

In RX measurement, port 1 of the network analyzer is the output and port 2 the input. The output port generates a continuous wave (CW) IF signal that is swept from $2.4 \mathrm{GHz}$ to $5 \mathrm{GHz}$, which is then up-converted in the reference RF unit to the OTA RF frequency of $26.4 \mathrm{GHz}$ to $29 \mathrm{GHz}$. Port 2 measures the IF signal coming from the DUT RF unit. The reference RF unit and the DUT are identical units. All antenna ports of the reference RF unit were active during the measurement in order to provide maximum signal-tonoise ratio. The DUT was controlled via remote connection so that only one antenna branch at a time is active and others are disabled. All 32 values of 5-bit phase shifters in RX and TX modes from all 16 branches were measured. Automated control code was created in Matlab for controlling the DUT and the measurement instrument and for collecting the measured S-parameters.

The number of S-parameter measurements for both RX and TX modes is 512 (16 antenna branches, each with 32 phase shifter values). By automating the OTA measurement, the measurement time was reduced to around 1 hour. This is much less compared with the conducted measurement, which was previously used to measure the phases.

\section{Data Post-processing}

After S-parameter files are collected, they are used to calculate the phase difference between antenna branches. One branch is selected as a reference, to which other branches are compared. Hence, the relative phase responses can be expressed as

$$
\theta_{i, j}=\angle\left(\frac{S_{21, i, j}}{S_{21, \mathrm{REF}}}\right),
$$

where $\theta_{i, j}$ denotes the phase response of $j$ th phase shifter value in $i$ th branch, $S_{21, i, j}$ is the OTA-channel measured with branch $i$ active with $j$ th phase control word, and $S_{21, \text { REF }}$ is 
TABLE I: RX and TX calibration values and phase control words for ideal and calibrated case in degrees for $+10^{\circ}$ steering.

\begin{tabular}{|c|c|c|c|c|c|c|c|c|c|c|c|c|c|c|c|c|}
\hline Branch & 1 & 2 & 3 & 4 & 5 & 6 & 7 & 8 & 9 & 10 & 11 & 12 & 13 & 14 & 15 & 16 \\
\hline Ideal phase control & 0.0 & 62.5 & 125.0 & -172.5 & -109.9 & -47.4 & 15.1 & 77.6 & 77.6 & 15.1 & -47.4 & -109.9 & -172.5 & 125.0 & 62.5 & 0.0 \\
\hline RX cal. value & -84.4 & -93.5 & -105.6 & -101.4 & -114.0 & -118.0 & -135.4 & -145.1 & -105.9 & -98.1 & -64.7 & -55.4 & -44.4 & -50.0 & -39.9 & 0.0 \\
\hline RX phase control & -84.4 & -31.0 & 19.5 & 86.1 & 136.0 & -165.5 & -120.3 & -67.5 & -28.0 & -83.0 & -112.1 & -165.3 & 143.2 & 75.1 & 22.6 & 0.0 \\
\hline TX cal. value & -35.1 & -61.2 & -70.9 & -41.2 & -74.9 & -82.3 & -92.3 & -104.6 & -22.8 & -53.9 & -24.0 & -7.2 & -19.2 & -33.8 & -26.5 & 0.0 \\
\hline TX phase control & -35.1 & 1.3 & 54.15 & 146.4 & 175.2 & -129.7 & -77.2 & -27.0 & 54.8 & -38.8 & -71.4 & -117.1 & 168.4 & 91.2 & 36.0 & 0.0 \\
\hline
\end{tabular}

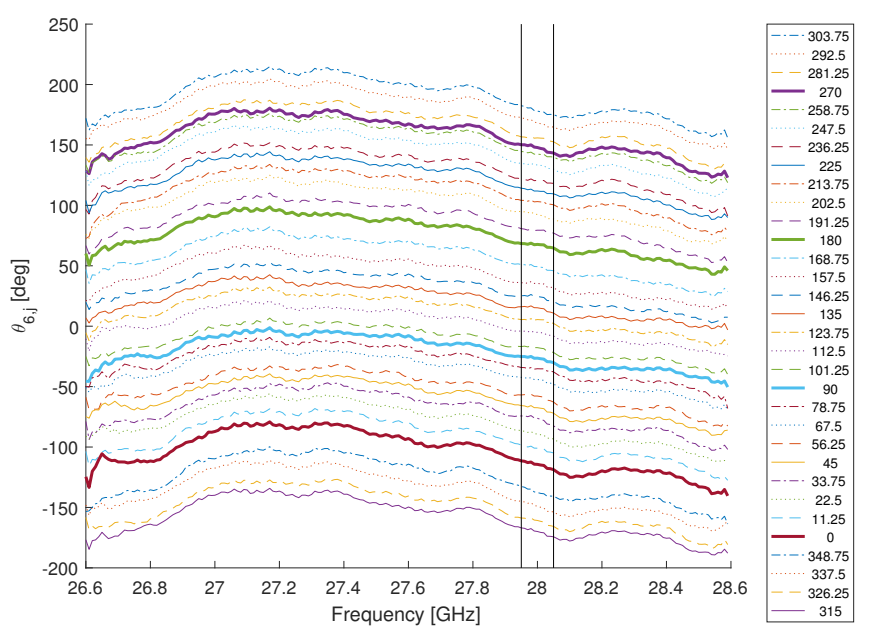

Fig. 3: RX side phase measurement results for branch 6 over different phase shifter values.

the OTA-channel of the reference branch with reference phase control. Expression (1) removes the effect of measurement devices and OTA channel. Hence, external calibration is not required. In our case, branch 16 with $0^{\circ}$ phase control was selected as a reference.

An example of the relative phase responses with different phase shifter values for branch 6 is presented in Fig. 3. In the figure, it is shown that there is frequency dependency in the normalized phase responses. Furthermore, there is also variation over the phase shifter values, i.e. not all phase steps are ideal $11.25^{\circ}$ steps. An example band of interest, $100 \mathrm{MHz}$ band around $28 \mathrm{GHz}$ is marked with black lines. The phase values are fluctuating inside the band. Therefore, the average phase over the frequency band is calculated. Hence, each band of interest can be calibrated separately by using the same measurement data. After compensating the ideal phase shift from the results, the average over phase shifter values is calculated and used for calibration.

\section{Calibration Procedure}

In Table I, phase excitation values to steer the main lobe beam to $+10^{\circ}$ azimuth angle is presented. The ideal values for the phase excitation are the same used in the uncalibrated case as phase shifter control words. In the calibration procedure, the measured calibration values are added to the ideal values to derive the calibrated phase shifter control words. Phase response of RX and TX are different, although they share the same phase shifters. Hence, depending on the operation

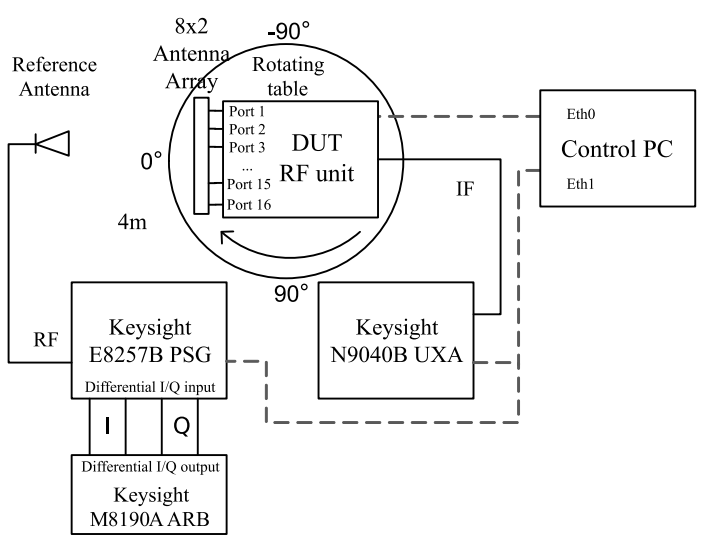

Fig. 4: RX side radiation pattern measurement setup.

mode of the RF unit different calibration parameters need to be used. This needs to be considered especially in Time-division duplexing (TDD) operation. Moreover, the system should be designed so that calibration values can be changed at the rate of TDD cycle.

\section{Radiation Pattern Measurements Using WIDEBAND 5G WAVEFORM}

The measured calibration values are verified by measuring radiation patterns with and without calibration. Measurement setup is presented in Fig. 4, where the RF unit is placed on a rotary table and rotated over azimuth half-plane of $\left[-90^{\circ},+90^{\circ}\right]$ with 1 degree steps. Traditionally radiation patterns are measured with a narrow $\mathrm{CW}$ signal. However, in our validation measurements we used a $100 \mathrm{MHz}$ wide $5 \mathrm{G}$ NR 64-QAM CP-OFDM signal [1] in order to better emulate the operation condition in which the unit is transmitting or receiving real modulated data. In RX measurement, 5G signal was generated with an arbitrary waveform generator (ARB) and upconverted to $28 \mathrm{GHz}$ RF frequency with PSG signal generator. A-info LB-28-15 standard gain horn antenna was used to radiate signal towards the DUT. UXA signal analyzer was used to measure the received signal over the $100 \mathrm{MHz}$ band at the IF frequency. TX measurements was also done by using the same procedure. For simplicity, only RX side radiation pattern measurements are presented in this paper. The radiation pattern measurements where automated by Matlabbased control software.

Results of the uncalibrated measurement are presented in Fig. 5a and calibrated measurement in Fig. 5b. Although in the uncalibrated scenario the phase excitations have some random 


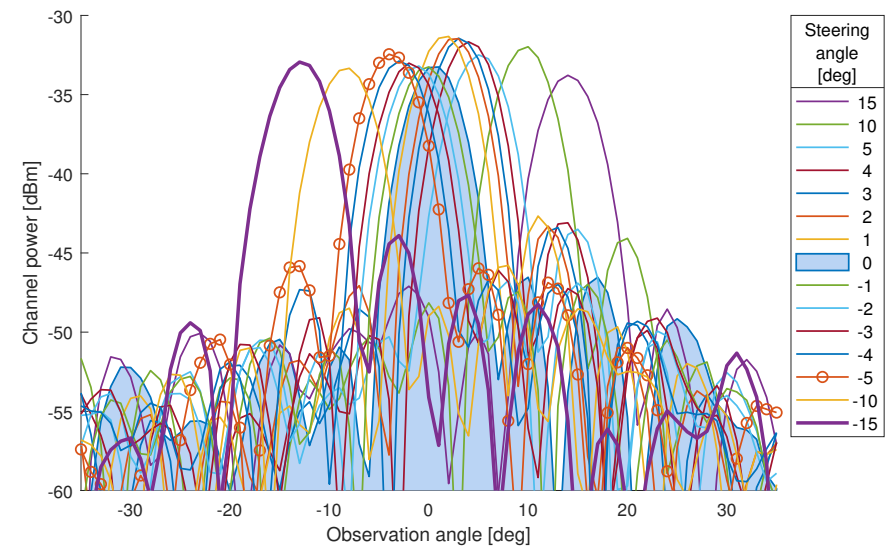

(a)

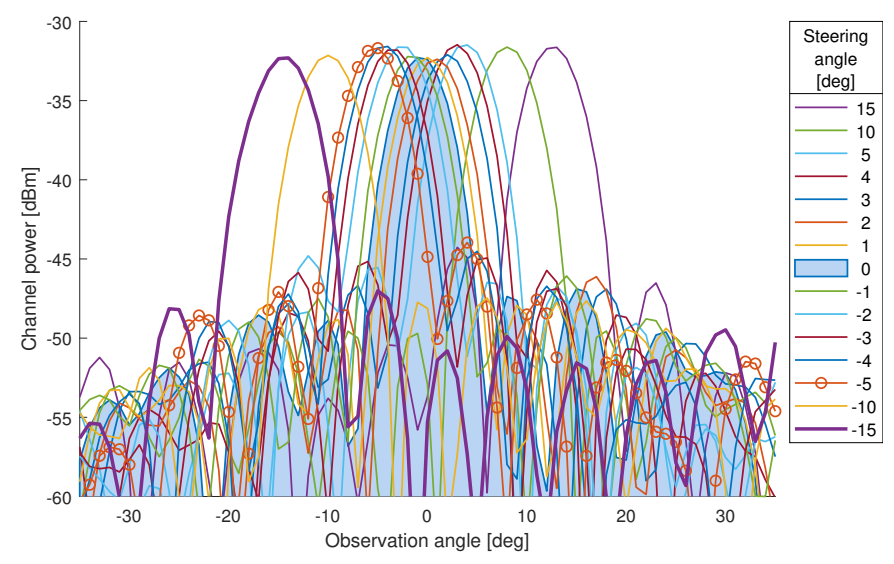

(b)

Fig. 5: RX radiation patterns on $28 \mathrm{GHz}$ RF frequency, measured with $100 \mathrm{MHz}$ wide 5GNR signal. Different steering angles are presented, with the measurement step size of $1^{\circ}$ : (a) uncalibrated, (b) calibrated.

device dependent factors, coarse beam steering is achieved. This is due to the fact that large enough antenna array is averaging parts of the phase errors out. In our measurement, the number of independent branches was 16 , which is enough to significantly reduce phase error due to averaging, but is not able to fully remove the impact. Furthermore, due to the fact that each RF branch drives a 4-element sub-array spaced with one wavelength [9], more errors can be tolerated compared with the half of a wavelength spaced array.

Measurement results show that the phase calibration changes the shape of the radiation patterns. The calibration stabilizes the amplitude variation over the steering angles. Variation of maximum main lobe amplitude in uncalibrated measurement is $2.45 \mathrm{~dB}$, which is reduced to $0.92 \mathrm{~dB}$ in calibrated measurement. Also, the average side lobe levels are reduced from $-12.5 \mathrm{~dB}$ down to $-14.1 \mathrm{~dB}$. In Fig. 5, the steering angle witch produces the worst side lobe level is presented for the uncalibrated case with bold line and for the calibrated case with circular markers. The beam pattern analysis was done with steering values from $-15^{\circ}$ to $15^{\circ}$, since larger steering angles produces grating lobes due to the large spacing between the smaller sub-arrays driven with independent RF chains in our example RF unit. Calibration does not have significant effect on half power beamwidth, which is from $5.3^{\circ}$ to $5.9^{\circ}$, having the smallest values for beams steered close to the boresight.

\section{CONCLUSion}

In this paper, OTA phase measurement setup and calibration method for $5 \mathrm{G} \mathrm{mmW} \mathrm{RF} \mathrm{unit} \mathrm{was} \mathrm{presented.} \mathrm{The}$ measurement procedure utilizes one branch as a reference and hence it does not require external calibration of the setup. The measurement takes into account all the inaccuracies from the RF implementation down to the physical antenna interface and antenna elements. Impact of calibration was verified by conducting radiation pattern measurements using a $100 \mathrm{MHz}$ wide 5G NR 64-QAM signal. OTA measurement was shown to improve the radiation pattern quality in terms of main lobe gain flatness and side lobe levels. Main lobe gain regularity was improved by $1.5 \mathrm{~dB}$ and the side lobe levels were reduced by $1.6 \mathrm{~dB}$ over the measured steering angles.

\section{ACKNOWLEDGMENT}

This work was supported by the Academy of Finland 6Genesis Flagship (grant no. 318927) and Business Finland funded Micro-operator concept for boosting local service delivery in 5G (uO5G) and 5G Finnish Open Research Collaboration Ecosystem (5GForce) projects.

\section{REFERENCES}

[1] (2018) 3GPP TS $38.104 \quad$ - Base Station (BS) radio transmission and reception (Release 15). [Online]. Available: https://portal.3gpp.org/desktopmodules/Specifications/ SpecificationDetails. aspx ? specificationId $=3202$

[2] T. S. Rappaport et al., "Millimeter Wave Mobile Communications for 5G Cellular: It Will Work!" IEEE Access, vol. 1, pp. 335-349, 2013.

[3] N. Tyler, B. Allen, and A. H. Aghvami, "Calibration of smart antenna systems: measurements and results," IET Microwaves, Antennas Propagation, vol. 1, no. 3, pp. 629-638, June 2007.

[4] R. Valkonen, "Compact 28-GHz phased array antenna for 5G access," in 2018 IEEE/MTT-S International Microwave Symposium - IMS, June 2018, pp. 1334-1337.

[5] M. E. Leinonen, G. Destino, O. Kursu, M. Sonkki, and A. Pärssinen, "28 GHz Wireless Backhaul Transceiver Characterization and Radio Link Budget," ETRI Journal, vol. 40, no. 15, pp. 89-100, Feb. 2018.

[6] O. Kursu et al., "Design and measurement of a $5 \mathrm{G} \mathrm{mmW}$ mobile backhaul transceiver at $28 \mathrm{GHz}$," EURASIP Journal on Wireless Communications and Networking, vol. 2018, no. 1, p. 201, Aug 2018. [Online]. Available: https://doi.org/10.1186/s13638-018-1211-5

[7] D. Yang, L. . Yang, and L. Hanzo, "DFT-Based Beamforming WeightVector Codebook Design for Spatially Correlated Channels in the Unitary Precoding Aided Multiuser Downlink," in 2010 IEEE International Conference on Communications, May 2010, pp. 1-5.

[8] C. A. Balanis, Antenna Theory Analysis And Design, 3rd ed. New Jersey, United States of America: John Wiley \& Sons, Inc., 2005.

[9] M. Sonkki et al., "Linearly Polarized 64-element Antenna Array for mmWave Mobile Backhaul Application," in 2018 12th European Conference on Antennas and Propagation (EuCAP)), London, UK, Apr. 2018.

[10] A. W. Mbugua, W. Fan, Y. Ji, and G. F. Pedersen, "Millimeter Wave Multi-User Performance Evaluation Based on Measured Channels With Virtual Antenna Array Channel Sounder," IEEE Access, vol. 6, pp. 12318-12326, 2018. 